\title{
Starch Pickering Emulsion: A Safe Vehicle for Topical Drug Delivery
}

\author{
By Joana Marto* \\ Luis Gouveia ${ }^{\dagger}$ \\ Lidia Goncalvest \\ Aida Duarte ${ }^{+}$ \\ Pedro Pinto \\ Teresa Cidade \\ Eduardo Oliveira \\ Antonio Almeida"
Helena Ribeiro
}

Pickering emulsions differ from classical emulsions, because the first are stabilized by solid particles instead of surfactants. This type of emulsions has been widely investigated in pharmaceutical and cosmetic fields since they present less adverse effects than the classical emulsions that are stabilized by surfactants. The present study was conducted in order to characterize physically and chemically a preservativefree and surfactant-free w/o emulsion for topical application and to evaluate its safety profile. Oscillatory and steady state shear measurements were performed for angular frequencies $(\omega)$ between 1 and $100 \mathrm{rads}-1$ and shear rates () between 1 and $1000 \mathrm{~s}-1$ at $25{ }^{\circ} \mathrm{C}$, and the emulsions were examined by brightfield light microscopy. The antimicrobial activity was performed according to a modification of membrane filtration method described in the Ph.Eur.. In order to predict the cutaneous irritation to the emulsion the cell viability was evaluated using $\mathrm{Df}$ and HaCaT cell lines in a MTT assay. A HRIPT was used to study the irritancy and sensitizing potential of emulsion in 53 volunteers. Rheological measurements indicated that the system is well structured. The microstructure is related to the rheological behaviour in relation to particle size, shape and distribution. The antimicrobial study demonstrates that this particular w/o emulsions present self-preservation properties. Cytotoxicity results showed that the emulsion can be considered non-irritant. And in vivo studies confirmed that both emulsions did not induce any irritative or allergic reactions, showing that this product show very good skin compatibility. This study confirms that starch Pickering emulsions present a well structure system with a safe profile and the use of appropriate excipients

${ }^{*}$ PhD Student, University of Lisbon, Portugal.

${ }^{\dagger}$ Assistant Professor, University of Lisbon, Portugal.

*Assistant Professor, University of Lisbon, Portugal.

${ }^{+}$Associate Professor, University of Lisbon, Portugal.

Assistant Professor, PhDTrails, Portugal.

- Cenimat/I3N, New University of Lisbon, Portugal.

Pharmacist, Laboratórios Atral S.A., Portugal.

^Full Professor, University of Lisbon, Portugal.

*Associate Professor, University of Lisbon, Portugal. 
allows preparing w/o emulsions with the obvious benefits of avoiding the drawbacks often associated with antimicrobial preservatives and surfactants agents.

Keywords: Pickering emulsions, starch, topical drug delivery

\section{Introduction}

Dosage forms for dermatological drug therapy are intended to produce desired therapeutic action at precise sites in the epidermal tissue. The drug's ability to penetrate the epidermis, dermis, and subcutaneous fat layers depends on the physicochemical properties of the drug, the carrier base and skin condition (Raposo et al., 2013a). Thus, one of the strategies currently being used to overcome these drawbacks is to develop novel drug delivery systems, such as nanoparticulate carriers, as well as to optimize conventional delivery systems.

Current pharmaceutical emulsions are mostly stabilized by synthetic surfactants that must be kept to a minimal because many can cause skin irritation and disrupt its barrier function. Synthetic surfactants can be intrinsically toxic or may alter the distribution and elimination of coadministered drugs (Bouyer et al., 2012). Consequently, solid-stabilized emulsions, the so-called Pickering emulsions, constitute an interesting strategy for encapsulation drugs in pharmaceutical formulation (Chen et al., 2011). Pickering emulsions are emulsions stabilized by solid particles instead of emulsifiers (Chevalier and Bolzinger, 2013). Many solid particles can be used for Pickering emulsions and they can be organic, such as polymer latex or starch, or inorganic, such as silica and clay particles (Kaewsaneha et al., 2013; Timgren et al., 2011). The stabilization of emulsion droplets by solid particles is possible due to their dual wettability. This allows for the spontaneous accumulation of particles at the oil-water interface and stabilizing it against coalescence by volume exclusion and steric hindrances (Matos et al., 2013; Fan and Striolo, 2012). Intact starch granules are a new source of particles for stabilizing emulsions. The use of these particles to stabilize emulsions has been attracting substantial research interest due to their distinctive characteristics and promising technological applications. These solid-stabilized emulsions avoid the application of hazardous surfactants and create a new application prospect of emulsion.

Pickering emulsions can be considered either as new dosage forms for topical drug delivery or as new targeting systems in cosmetic products. This paper reports the stabilization ability of starch granules and its application on pharmaceutical and cosmetic emulsions. 


\section{Materials and Methods}

Materials

Paraffin was obtained from Mosselman (Belgium) and Miglyol ${ }^{\circledR} 812$ (caprylic/capric acid triglyceride), was kindly gifted by Sasol (Germany). The starch used was modified starch obtained from AkzoNobel (Sweden). Purified water was obtained by inverse osmosis (Millipore, Elix 3) which was then sterilized and filtrated.

\section{Emulsion Processing}

Two surfactant-free w/o emulsions stabilized by starch (w/o) were developed. The disperse phase was purified water, the continuous phase consisted on EA (lipophilic emollient - triglycerides) or LP (lipophilic occlusive - hydrocarbons) and the solid particle was starch. To prepare the emulsion, the starch was first dispersed in the oil phase using a vortex disperser until total dispersion of the starch. The oil and aqueous phases of the emulsion were then mixed together with an UltraTurrax ${ }^{\circledR}$ T25 homogeniser (IKA®Werke GmbH \& Co. KG, Germany).

Microscopy and Droplet Size Analysis

A computerized image analysis device coupled to an Olympus microscope (Olympus BX51, Germany), was used for the microscopic observations, in bright field to determine emulsions' droplet size. Samples were examined one day after preparation. Droplet samples were photographed and slides were made so that droplets $(\mathrm{n} \geq 625)$ could be measured (British Standard Institution 1990). The droplet size was statistically analysed in order to assess the size distribution and to determine the geometric standard deviation $(\sigma \mathrm{g})$.

\section{Rheological Analysis}

The rheological measurements were obtained using a Bohlin Gemini HRNano Rheometer (Malvern, UK) using cone and plate geometry (cone angle $2.0^{\circ}$ and radius $20 \mathrm{~cm}$ ), at $25^{\circ} \mathrm{C}$. All samples were tested 1 month after preparation and storage at $25^{\circ} \mathrm{C}$, at least in triplicates using fresh samples. Steady state shear measurements were performed for shear rates $(\dot{\gamma})$ between 1 and $1000 \mathrm{~s}^{-1}$. Oscillation frequency sweep tests were performed over a frequency range of 1 to $100 \mathrm{rad} \mathrm{s}^{-1}$.

\section{Antimicrobial Activity}

The antimicrobial activity was performed according to a modification of membrane filtration method described in the $\mathrm{Ph}$. Eur. (5.1.3.) (European Pharmacopeia, 2009). Briefly, 8 samples of $20 \mathrm{~mL}$ were prepared separately, and afterwards contaminated with an inoculum of $10^{8}$ microorganisms per millilitre of Pseudomonas aeruginosa ATCC 9027, Staphylococcus aureus ATCC 6538, Candida albicans ATCC 10231 and Aspergilus niger ATCC 16404. 
The antimicrobial activity of the emulsions was evaluated during 28 days. Results were expressed as the log reduction of the cfu at $0 \mathrm{~h}, 48 \mathrm{~h}, 7$ days, 14 days and 28 days.

The bacteria should be diminished at least by about 2 log-steps after two days, by about $3 \log$-steps after 7 days and on day 28 their number must not be increased. In the case of fungi, the cfu should be reduced at least about 2 logsteps after 14 days and on day 28 the colony numbers should be not increased for A criteria and $1 \log$-step after 14 days and on day 28 the colony numbers should be not increased for B criteria.

\section{In vitro Cytotoxicity Studies}

\section{Cell Culture Conditions}

Human adult dermal fibroblasts cells Df (ZenBio, Inc., USA), and a spontaneously immortalized human keratinocyte cell line, HaCaT, (CLS, Germany) were grown in RPMI-1640 ${ }^{\circledR}$ (Gibco, UK) medium supplemented with $10 \%(w / V)$ fetal bovine serum (FCS, Life Technologies, Inc., UK), penicillin $(100 \mathrm{IU} / \mathrm{mL})$, and streptomycin $(100 \mu \mathrm{g} / \mathrm{mL})$ in a humidified $95 \%$ $\mathrm{O}_{2} / 5 \% \mathrm{CO}_{2}$ environment at $37{ }^{\circ} \mathrm{C}$. For the subculture, cells growing as a monolayer were detached from the tissue flasks by treatment with $0.05 \%(\mathrm{w} / \mathrm{V})$ trypsin/EDTA (Invitrogen, UK). The viability and cell count were monitored routinely using Trypan blue dye exclusion method (Strober, 2001).

\section{Cytotoxicity Assays}

To determine in vitro emulsions effect on cell viability, cells (cultured in 96-well microplates) were incubated with $10 \mu \mathrm{l}$ of the emulsions for $24 \mathrm{~h}$ and cell viability was determined with the MTT assay. After the time of exposition, medium was replace by $0.3 \mathrm{mM}$ PI in culture medium (stock solution $1.5 \mathrm{mM}$ in DMSO, diluted with culture medium 1:5000). Fluorescence was measured (excitation, $485 \mathrm{~nm}$; emission, $590 \mathrm{~nm}$ ) in microplate reader (FLUOstar Omega, BMGLabtech, Germany), and then, the MTT assay was performed. Medium was replaced by medium containing $0.25 \mathrm{mg} / \mathrm{mL}$ MTT. The cells were further incubated for $3 \mathrm{~h}$. On the plates that contain reduced MTT, the media was removed and the intracellular formazan crystals were solubilized and extracted with $100 \mu \mathrm{l}$ DMSO. After $15 \mathrm{~min}$ at room temperature, the absorbance was measured at $570 \mathrm{~nm}$ in a microplate reader (FLUOstar Omega, BMGLabtech, Germany). The relative cell viability (\%) compared to control cells was calculated for the MTT assay by:

$$
\text { Eq. } 3-\% \text { cell viability }=\frac{\text { Abssample }}{\text { Abscontrol }} \times 100
$$

where $\mathrm{Abs}_{\text {sample }}$ is the absorbance value obtained for cells treated with nanoparticles and $\mathrm{Abs}_{\text {control }}$ is the absorbance value obtained for cells incubated with culture medium.

Within an experiment, each condition was assayed in triplicate and every experiment was performed at least three times, with a total of nine replicates. 
The percentage of viable cells was established relatively to cells treated with emulsions.

\section{Human Repeated Insult Patch Test}

A safety evaluation study was performed on emulsions, using a Marzulli \& Maibach (Marzulli and Maibach, 1976) HRIPT protocol. In brief, the emulsions were applied on the back of 53 healthy volunteers that gave their informed written consent. For the induction period, a series of nine patches (Finn Chamber standard) were performed over a period of 3 weeks. At the product site, an occlusive patch containing $20 \mathrm{mg}$ of the formulation was applied to the left side of the back where it remained for 48 hours. After that period, the patch was removed, the skin was evaluated and a new patch was applied. Reactions after patching were scored according to International Contact Dermatitis Research Group (Fregert and Bandmann, 1975).

A 2 week rest period was observed without application of the test material. During the challenge period, new patches were prepared and fixed in the same manner as in the induction period, but also on the right side of the back (i.e., a blank site). The patches were removed after 48 hours and scoring of skin reactions was performed in the same manner as before at 48, 72, and 96 hours after patching using the same International Contact Dermatitis Research Group scoring system. The protocol was approved by the local Ethical Committee and respected the Helsinki Declaration and the AFSSAPS regulations on performed HRIPT studies on cosmetic products. The study was conducted under the supervision of a dermatologist who participated in the evaluation of irritation/allergic reactions to the emulsions.

\section{Results and Discussion}

Microscopy and Droplet Size Analysis

The light microscopy images and the plot of the particle size distribution revealed that the size of droplets and the microstructure of the systems depended on the lipid used (Figures 1 and 2). 
Figure 1. a) Photomicrographs of Emulsion EA (Magnification 100x) and b) Droplet Size Distribution of Emulsion EA. Median Particle Size $=125.2 \mu \mathrm{m}$ and $\sigma g=188$.

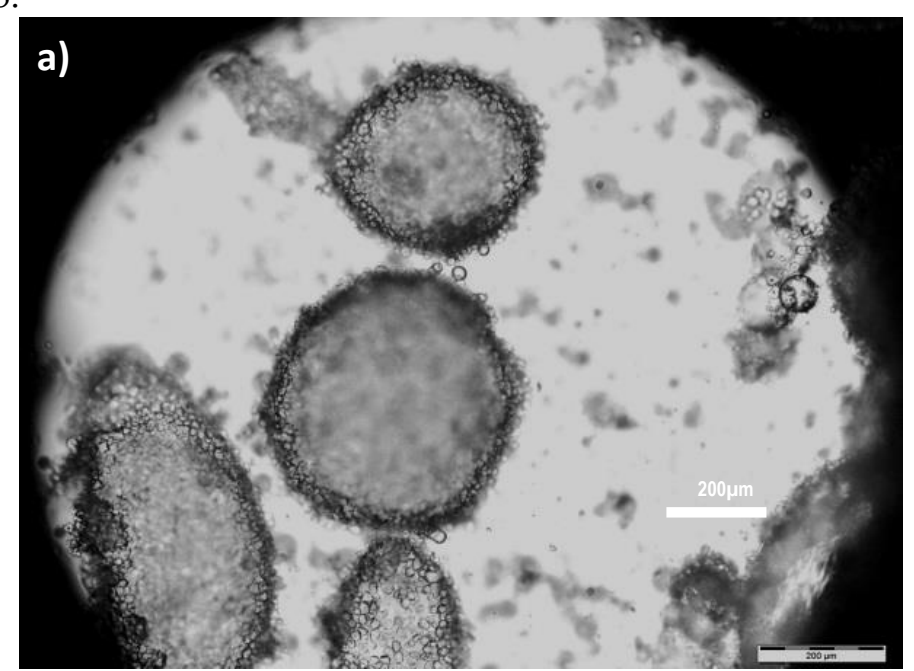

b)

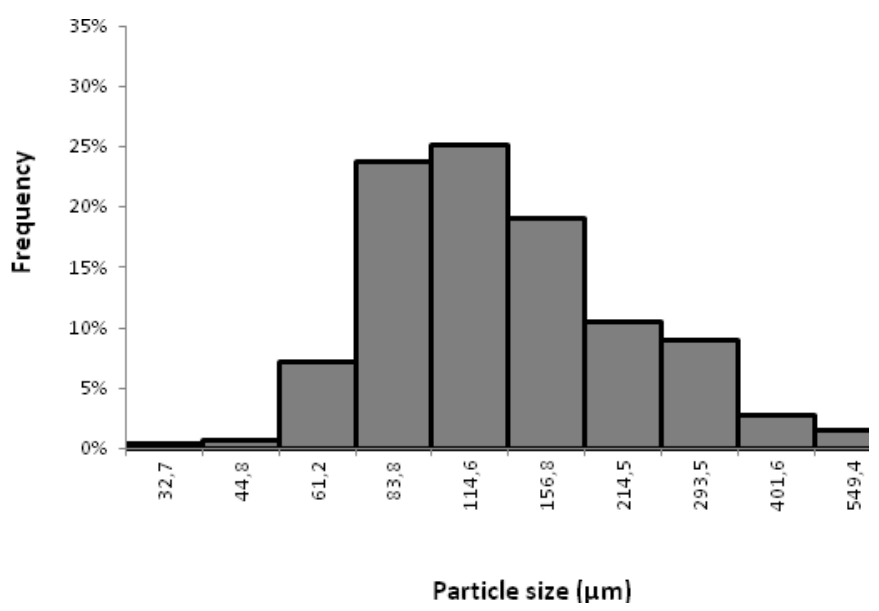

In the case of emulsion EA bigger inner droplets with a non-homogeneous size were observed (Figure 1). Emulsion EB presented several small inner droplets of water in the oil phase. The droplets presented a symmetric and a well defined microstructure (Figure 2). Droplet size differences between these emulsions are probably due to affinity of the lipids for the internal phase. Burkhardt et al. (2009) proved that a correlation exists between the polarity of the solvent and the amount of emulsifier required for stabilization, the more polar solvents in general requiring larger amounts of emulsifier, which is the case of EA, in order to decrease de droplet size distribution. 
Figure 2. a) Photomicrographs of Emulsion EB (Magnification 100x) and b) Droplet Size Distribution of Emulsion EB. Median Particle Size $=103.3 \mu \mathrm{m}$ and $\sigma g=180$.

b)
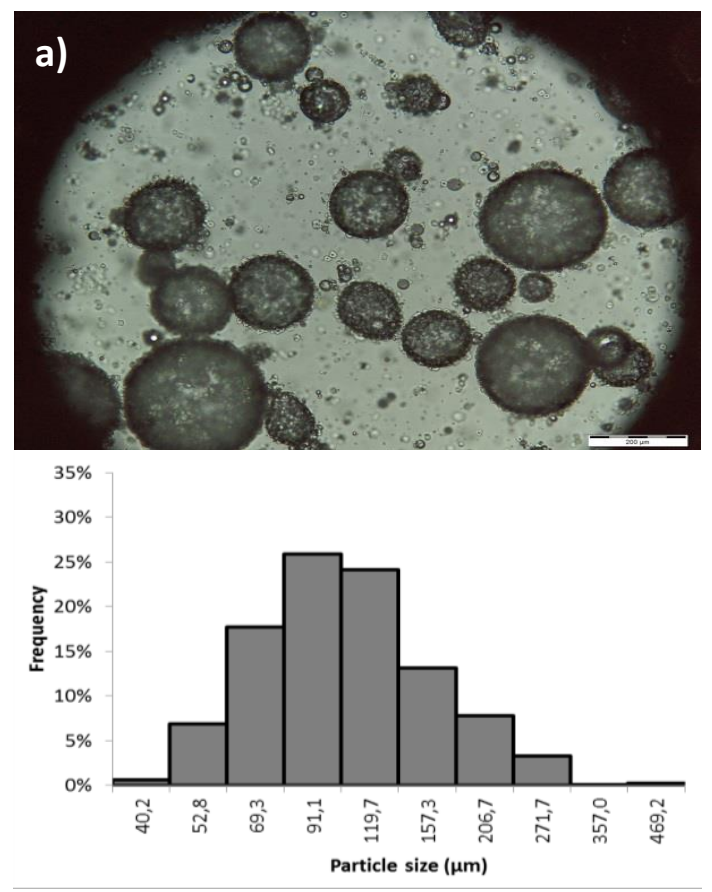

Rheological Analysis

Continuous shear experiments measure the ability of each system to resist structural breakdown during the standardized shearing procedure. Representative flow curves are shown in Figure 3 for both emulsions at the same temperature.

Figure 3. Viscosity as Function of Shear Rate for Both Emulsions at $25^{\circ} \mathrm{C}$.

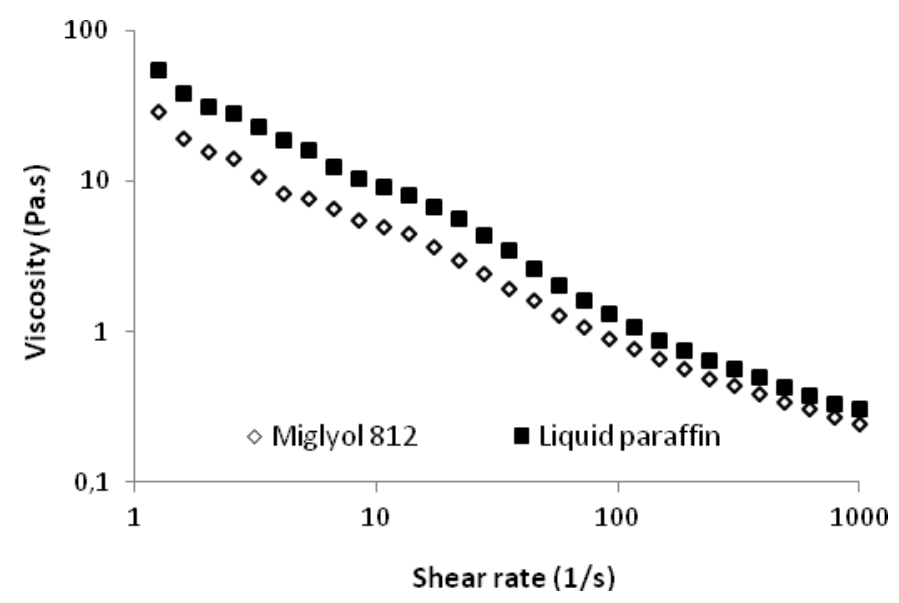

The steady state shear viscosity decreases with the increase of the shear rate, showing a shear-thinning behaviour for both emulsions. 
In order to obtain information about viscoelastic behaviour of the investigated systems and the network structure formed by particle-particle interactions, an oscillation frequency sweep test was conducted at a strain in the linear viscoelastic regime, previously determined.

The viscoelastic properties are expressed with the following dynamic parameters: (1) storage modulus, $\mathrm{G}^{\prime}(\mathrm{Pa})$, characterizing the elastic behaviour and representing the energy stored by the system; (2) loss modulus, G" (Pa), characterizing the viscous (plastic) behaviour, representing the energy dissipated by the system (Gašperlin et al., 1998; Brummer, 2006; Raposo et al., 2013b).

For the two emulsions, $G^{\prime}$ is must higher than $G^{\prime \prime}$, meaning a dominant elastic behaviour of both emulsions. Also, in both cases, the slope of the curve $G^{\prime}$ vs. $\omega$ is very small meaning that both systems are well structured. As expected, the $G^{\prime}$ and $G$ ', increased with temperature, probably due to the onset gelatinization of the polymer that stabilized the inner droplets. Figure 4 indicates that EB presents higher G' and G' moduli than EA.

These findings are in accordance through the previous results of droplet size and distribution, emphasizing the influence of lipids in the structure of these emulsions. The emulsion containing hydrocarbons presented a slightly stronger and organized structure.

Figure 4. Storage and Loss Modulus as a Function of Angular Frequency for Both Emulsions at $25^{\circ} \mathrm{C}$.

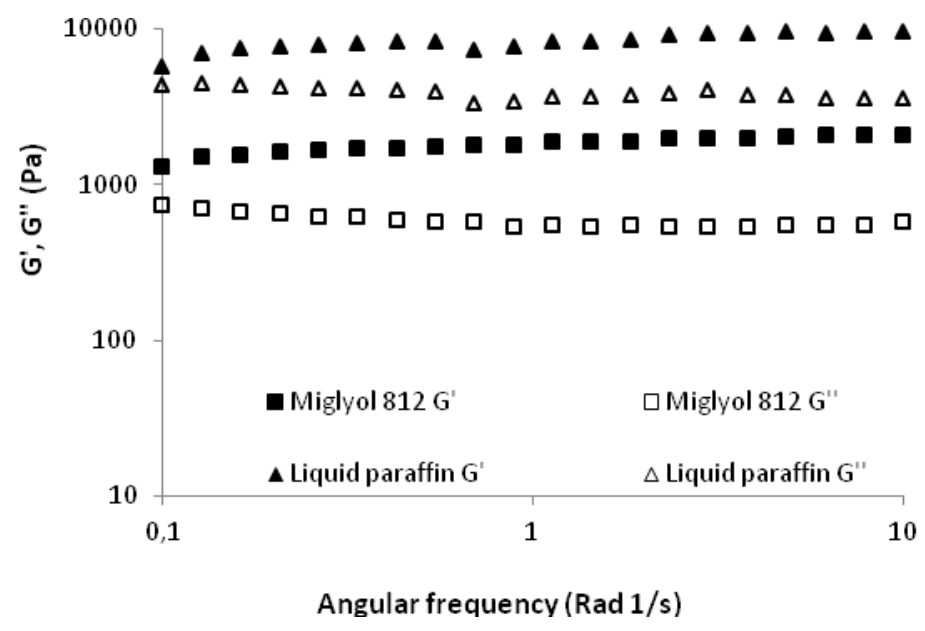

\section{Antimicrobial Activity}

There are many cosmetic ingredients, such as preservatives and fragrances, recognized to induce adverse effects, however, a wide variety of skin care products contain preservatives (Lee et al., 2007). Consequently, it is important the development of new preservative-free and self-preserving cosmetics.

Emulsions showed to be effective against the tested bacteria and fungi. For bacteria ( $P$. aeruginosa and $S$. aureus) a decrease in the microbial count (cfu) was found after the first $48 \mathrm{~h}$ and for $C$. albicans and A. niger a $2 \log$ reduction was noticed after 7 days, followed by undetectable cfu for the subsequent 
observation days. Thus, both emulsions complied with the A criterium for bacteria and fungi and B criterium for yeasts. The emulsions revealed an antimicrobial activity which conformed to the requirements of the preservation efficacy test for topical formulations according to Ph. Eur..

\section{In vitro Cytotoxicity Studies}

To investigate the potential cytotoxicity of the w/o Pickering emulsions, the cell viability was evaluated using DF and HaCaT cell lines in a MTT assay. It was found that the two emulsions were not cytotoxic in both DF and $\mathrm{HaCaT}$ cells on the concentration tested.

Additionally, emulsions stabilized by solid particles can be distinguished from emulsions stabilized with "traditional" emulsifiers concerning their irritative potential, for the reason that emulsifiers have the potential to act as penetration enhancers by conditional decreasing the SC and hence may enable or enhance diffusion of other molecules through the skin (Djekic and Primorac, 2008; Bos and Meinardi, 2000). Moreover, according to many authors, surfactants cause irritant reactions when applied to the skin, due to their ability to solubilize lipid membranes (Effendy and Maibach, 1995; Wilhelm et al., 1994).

On the other hand, the starch granules, due to their high molecular weight (> $10000 \mathrm{Da}$ ), are not able to penetrate the SC (Bos and Meinardi, 2000; Raposo et al., 2013c).

Moreover, according to the OECD guideline, an irritant substance is predicted if the mean relative tissue viability is found below $50 \%$ of the mean viability of the negative controls for a 15-60 min exposition time (OECD Test No. 439. 2010). In the present assay, cells were exposed to test samples for 24 $\mathrm{h}$ with the cell viability above $50 \%$. The absence of skin-irritant effects at the concentrations tested indicated that emulsions could be safe for topical use.

\section{Human Repeated Insult Patch Test}

Under experimental conditions adopted repeated applications of emulsions in occlusive patch with a panel of 53 volunteers, did not induce any irritant reaction, showing that these emulsions show good skin compatibility (Induction phase). Moreover, the repeated applications did not induce any allergic reactions (Challenge phase).

\section{Conclusions}

In this work were produced w/o Pickering emulsions stabilized by starch particles. This study confirmed that starch-based Pickering emulsions present a well structure system for topical application with an acceptable profile. The use of appropriate excipients allows preparing w/o emulsions with the obvious advantages of avoiding the adverse effects often associated with antimicrobial preservatives and surfactants. 
Pickering emulsions stabilized by starch granules had good organoleptic characteristics and other properties that make them worthy to be used in topical products, as cosmetics and also as pharmaceutical forms.

\section{Acknowledgments}

This work is supported by the Ministry of Science (FCT-MCTES, SFRH/BDE/51599/2011), the strategic projects Pest-OE/SAU/UI4013/2011 and Pest-C/CTM/LA0025/2011- CENIMAT/I3N and Laboratórios Atral S.A., Portugal.

\section{References}

Bos, J.D. and Meinardi, M.M. 2000. The 500 Dalton rule for the skin penetration of chemical compounds and drugs. Experimental Dermatology. 9(3): p. 165-9.

Bouyer, E., Mekhloufi, G., Rosilio, V., Grossiord, J.L. 2012. Proteins, polysaccharides, and their complexes used as stabilizers for emulsions: Alternatives to synthetic surfactants in the pharmaceutical field? International Journal of Pharmaceutics. 436(1-2): 359-378

Brummer, R. 2006. Rheology Essentials of Cosmetic and Food Emulsions, Springer, Germany

Burkhardt, M., Kinzel, S., and Gradzielski, M. 2009. Macroscopic properties and microstructure of HSA based organogels: Sensitivity to polar additives. Journal of Colloid and Interface Science. 331(2): 514-521.

BS 1377: 1990 British Standard Institution. Part 2.

Chen, J., Vogel, R., Werner, S., Heinrich, G., Clausse, D., Dutschk, V. 2011. Influence of the particle type on the rheological behaviour of Pickering emulsions. Colloids and Surfaces A: Physicochemical and Engineering Aspects. 382(1-3): 238-245

Chevalier, Y. and Bolzinger, M.A. 2013. Emulsions stabilized with solid nanoparticles: Pickering emulsions. Colloids and Surfaces A: Physicochemical and Engineering Aspects. 439: 23-34

Djekic, L. and Primorac, M. 2008. The influence of cosurfactants and oils on the formation of pharmaceutical microemulsions based on PEG-8 caprylic/capric glycerides. International Journal of Pharmaceutics. 352(1-2): 231-9.

Effendy, I. and Maibach, H.I. 1995. Surfactants and experimental irritant contact dermatitis. Contact Dermatitis. 33(4):217-25.

European Pharmacopeia 7.0. 2009. Section 5.1.3. 528-529

Fan, H. and Striolo, A. 2012. Mechanistic study of droplets coalescence in Pickering emulsions. Soft Matter. 8: 9533

Fregert, S. and Bandmann, H-J. 1975. International Contact Dermatitis Research Group patch testing. New York: Springer-Verlag. 8-10

Gašperlin, M., Tušar, L., Tušar, M., Kristl, J., Šmid-Korbar, J.1998. International Journal of Pharmaceutics. 168, 243-254

Kaewsaneha, C., Tangboriboonrat, P., Polpanich, D., Eissa, M., Elaissari, A. 2013. Preparation of Janus colloidal particles via Pickering emulsion: An overview. Colloids and Surfaces A: Physicochemical and Engineering Aspects. 439: 35-42 
Lee, E., An, S., Choi, D., Moon, S., Chang, I. 2007. Comparison of objective and sensory skin irritations of several cosmetic preservatives. Contact Dermatitis. 56(3):131-6.

Marzulli, F.N. and Maibach, H.I. 1976. Contact allergy: predictive testing in man. Contact Dermatitis. 2:1-17

Matos, M., Timgren, A., Sjöö, M., Dejmek, P., Rayner, M. 2013. Preparation and encapsulation properties of double Pickering emulsions stabilized by quinoa starch granules. Colloids and Surfaces A: Physicochemical and Engineering Aspects. 423: 147-153

OECD Test No. 439. 2010. In vitro skin irritation: reconstructed Human epidermis test method, OECD guidelines for the testing of chemicals 1:1-18.

Raposo, S.C., Simões, S.D., Almeida, A.J., Ribeiro, H.M. 2013a. Advanced systems for glucocorticoids' dermal delivery. Expert Opinion on Drug Delivery. 10(6):857-77.

Raposo, S., Salgado, A., Eccleston, G., Urbano, M., Ribeiro, H.M. 2013b. Cold processed oil-in-water emulsions for dermatological purpose: formulation design and structure analysis. Pharmaceutical Development and Technology.19(4):41729

Raposo, S., Salgado, A., Gonçalves, L., Pinto, P.C., Urbano, M., Ribeiro, H.M. 2013c. Safety Assessment and Biological Effects of a New Cold Processed SilEmulsion for Dermatological Purpose. BioMed Research International. 2013:10.

Strober, W. 2001. Trypan Blue Exclusion Test of Cell Viability, in: Current Protocols in Immunology, John Wiley \& Sons, Inc.

Timgren, A., Raynr, M., Sjöö, M., Dejmek, P. 2011. Starch particles for food based Pickering emulsions. International Congress of Engineering and Food, Athens, Greece. Procedia Food Science. 1: 95-103

Wilhelm, K.P., Freitag, G., Wolff, H.H. 1994. Surfactant-induced skin irritation and skin repair. Evaluation of the acute human irritation model by noninvasive techniques. Journal of the American Academy of Dermatology. 30(6):944-9. 
